\title{
Prevalência e Aspectos Clínicos da Associação de Diabetes Melito Tipo 1 e Doença Celíaca
}

\begin{abstract}
RESUMO
Objetivos: Verificar a prevalência da associação entre diabetes melito tipo 1 (DM1) e doença celíaca (DC) e a presença de sintomas da DC, a ocorrência de outras doenças auto-imunes entre os pacientes e seus parentes de primeiro grau e as possíveis influências da DC no controle do diabetes. Métodos: Estudo transversal com 195 pacientes com DM1, que responderam ao questionário sobre a presença de sintomas gastrintestinais e a ocorrência de doenças auto-imunes em familiares. Foi dosada a IgA sérica e pesquisado o anticorpo antiendomísio (EMA). Os pacientes com EMA positivo foram submetidos à biópsia intestinal. Aqueles com DC confirmada por biópsia (grupo-casos) foram pareados com os pacientes apenas diabéticos (grupo-controle), de acordo com a idade ao diagnóstico de diabetes, o tempo de duração da doença e o gênero. Resultados: O EMA foi positivo em nove pacientes. Em sete a biópsia confirmou DC (prevalência de 4\%). No pareamento de casos (DM1 e DC) e controles (somente DM1), os sintomas gastrintestinais foram significativamente mais freqüentes no grupo casos, não sendo observada diferença com a ocorrência de doenças auto-imunes entre os parentes de primeiro grau e com o controle do diabetes (z peso, z estatura, dose de insulina e HbA1c). Conclusões: A prevalência de DC neste grupo de pacientes com DM1 foi de $4 \%$. A amostra de pacientes celíacos apresentou predomínio de sintomas gastrintestinais, porém a presença de DC não interferiu no controle do diabetes. (Arq Bras Endocrinol Metab. 2008; 52/4:635-641)
\end{abstract}

Descritores: Doença celíaca; Diabetes melito tipo 1; Prevalência; Pediatria; Doenças auto-imunes.

\begin{abstract}
Prevalence and Clinical Aspects When it Comes to the Association Between Type 1 Diabetes Mellitus (DM1) and Celiac Disease.

Objectives: To estimate the prevalence of type 1 diabetes mellitus (DM1) and celiac disease association and to verify the existence of celiac disease symptoms, as well as the occurrence of other autoimmune diseases among the patients, their first-degree relatives and the possible influences of celiac disease in diabetes control. Methods: It was done a cross-sectional study with 195 patients that answered a questionnaire about gastrointestinal symptoms and the occurrence of autoimmune diseases in their first-degree relatives. IgA was measured and antiendomysial antibody (EMA) was screened. The patients with positive EMA were submitted to intestinal biopsy. Those with celiac disease confirmed by biopsy (case group) were paired with DM1 patients without celiac disease (control group) according to age on diabetes diagnosis, diabetes duration and gender. Results: EMA was positive in nine patients. In seven of them the biopsy has confirmed celiac disease $(4.0 \%)$. Comparing the cases with controls, the gastrointestinal symptoms were significantly more frequent in the first group, but there was no difference between the groups regarding to the occurrence of autoimmune disease among the first-degree relatives and regarding to the control of diabetes ( $\mathrm{z}$ weight, $\mathrm{z}$ height, insulin dose, $\mathrm{HbA} 1 \mathrm{c})$. Conclusions: The prevalence found was $4.0 \%$. This sample of celiac patients showed a predominance of gastrointestinal symptoms, although the celiac disease did not influence the diabetes control. (Arq Bras Endocrinol Metab 2008; 52/4:635-641)
\end{abstract}

Keywords: Celiac disease; Diabetes mellitus type 1; Prevalence; Pediatrics; Autoimmune diseases. artigo original

\author{
FÁtIMA C. F. WHITACKER \\ GABRIEL HESSEL \\ Sofia H. V. Lemos-MarinI \\ Maria F. V. M. PAULINo \\ WALTER J. MinicucCl \\ GIL GuerRa-JúNIOR
}

Departamento de Pediatria da Faculdade de Ciências Médicas da Universidade Estadual de Campinas (Unicamp), SP, Brasil. 


\section{INTRODUÇÃO}

DIABETES MELITO TIPO 1 (DMl) caracteriza-se por hiperglicemia crônica resultante de insulinopenia progressiva por causa da destruição das células- $\beta$ pancreáticas por auto-anticorpos. Acomete, preferencialmente, crianças e adolescentes geneticamente predispostos (1).

Ainda que o desencadeamento desta auto-imunidade não esteja completamente esclarecido, o seu reconhecimento permitiu compreender a associação com outras doenças auto-imunes (DAI), entre elas a doença celíaca (DC), uma enteropatia imunomediada induzida pelo glúten, que apresenta genes de suscetibilidade comuns ao DMl, sobretudo os alelos DQAl*0501 e $\mathrm{DQB1}{ }^{*} 0201(2)$.

A descoberta de marcadores sorológicos permitiu que indivíduos assintomáticos, mas considerados de risco para DC, como os diabéticos, pudessem ser selecionados para biópsia intestinal, método considerado o padrão-ouro para o diagnóstico da DC (3). Dessa forma, a triagem sorológica contribuiu para aumentar significativamente a prevalência de DC, tanto na população em geral quanto nos grupos de risco (4).

$\mathrm{Na}$ Europa, a prevalência de DC entre os diabéticos varia muito entre os diversos países, atingindo até 16,4\%, com média de 4,1\% (5). No Brasil, os estudos são escassos (6-9) e em muitos serviços a investigação da DC ainda não foi incorporada à rotina de seguimento dos pacientes com DMl.

A importância do diagnóstico da DC está na oportunidade de se instituir precocemente o tratamento com o intuito de prevenir as possíveis complicações da DC não tratada, como baixa estatura, osteoporose, linfoma intestinal, entre outras (10). Em relação aos diabéticos, não há consenso de que a DC possa interferir negativamente no controle do diabetes.

Assim, este estudo teve por objetivos avaliar a prevalência da DC em um grupo de pacientes com DMl, pesquisar a presença de sintomas gastrintestinais sugestivos de DC e a ocorrência de outras DAI entre os pacientes e seus parentes de primeiro grau, bem como avaliar as possíveis interferências da DC no controle metabólico do diabetes.

\section{MÉTODO}

Trata-se de um estudo transversal realizado no Ambulatório de Diabetes do Departamento de Pediatria do
Hospital das Clínicas (HC) da Universidade Estadual de Campinas (Unicamp), no período de março de 2003 a março de 2004. Foram incluídos todos os pacientes com diagnóstico prévio de $\mathrm{DMl}$, idade superior a 2 anos, e que se encontravam em acompanhamento ambulatorial, totalizando, inicialmente, 195 pacientes. No dia de sua consulta de rotina, os pacientes e/ou seus responsáveis receberam informações detalhadas sobre o estudo, presentes no Termo de Consentimento Livre e Esclarecido (TCLE) aprovado pelo Comitê de Ética em Pesquisa da Faculdade de Ciências Médicas da Unicamp (Parecer n $\left.{ }^{\circ} 159 / 1998\right)$.

\section{Avaliação clínica}

Foram pesquisados sintomas clássicos ou do trato digestório (diarréia, distensão ou dor abdominal, flatulência, epigastralgia, vômitos e constipação intestinal crônica) e inespecíficos ou fora do trato digestório (artralgia, artrite, cefaléia, fadiga e crises convulsivas não relacionadas à hipoglicemia). As respostas foram consideradas afirmativas quando o paciente ou seu responsável considerava freqüente a ocorrência do(s) sintoma(s) em qualquer fase da vida, seja antes do início do DMl, ao diagnóstico, durante a evolução desta doença e/ou contemporâneo à entrevista. De acordo com a resposta, o paciente foi classificado como assintomático, sintomático com sintomas clássicos ou sintomático com sintomas inespecíficos. Quando referiu sintomas clássicos e inespecíficos foi classificado como sintomático clássico.

Os pacientes também foram interrogados quanto à presença de DAI em seus parentes de primeiro grau. As doenças pesquisadas foram: $\mathrm{DMl}, \mathrm{DC}$, doenças da tireóide, vitiligo, psoríase, lúpus eritematoso sistêmico e artrite reumatóide.

Do prontuário do paciente foram resgatados os antecedentes pessoais de DAI e os outros dados, como idade do diagnóstico do DMl, início do seguimento no Ambulatório de Diabetes do HC da Unicamp, valores de $\mathrm{HbAlc}$ do ano que antecedeu a pesquisa e dose de insulina no momento da avaliação. Todos os pacientes incluídos no estudo foram medidos e pesados e estes dados transformados em $\mathrm{z}$ escore, tomando-se como base os dados do National Center of Health Statistics (NCHS) de 2000.

\section{Avaliação laboratorial}

Foram coletados pelo Laboratório de Patologia Clínica do HC da Unicamp cerca de $8 \mathrm{~mL}$ de sangue venoso 
em tubo sem anticoagulante. Parte deste material destinou-se à dosagem de IgA por nefelometria (limite inferior de detecção de $23,5 \mathrm{mg} / \mathrm{dL}$ ). O restante do material foi encaminhado ao Laboratório de Gastroenterologia Pediátrica do HC da Unicamp, onde foi realizada a pesquisa do anticorpo antiendomísio (EMA) pelo método da imunofluorescência indireta, utilizando como substrato cordão umbilical humano cedido pelo Centro de Atenção Integral à Saúde da Mulher (CAISM) da Unicamp. Os soros que apresentaram fluorescência na diluição igual ou superior a 1:10 foram considerados positivos.

Em uma segunda etapa, os pacientes com EMA positivo foram submetidos à biópsia duodenal para confirmação diagnóstica. Este procedimento coube à equipe de Gastroenterologia Pediátrica do HC da Unicamp, sendo realizado por cápsula de Watson ou por endoscopia digestiva alta. Os fragmentos de biópsia foram analisados pelo Serviço de Anatomia Patológica do Gastrocentro da Unicamp e a caracterização histológica obedeceu à classificação de Marsh (11). Os pacientes com diagnóstico de DC confirmado foram orientados a seguir dieta isenta de glúten. Cada caso confirmado com a associação DC e DMl foi pareado a três casos que só apresentavam DMl, de acordo com o gênero, idade na realização da triagem e tempo de duração do diabetes.

\section{Análise estatística}

O programa SPSS versão 11.0 foi utilizado para o arquivo e o processamento dos dados. As variáveis contínuas foram descritas por média, desvio-padrão e valores mínimo e máximo e as variáveis categóricas por sua freqüência em porcentagens. $\mathrm{O}$ teste não-paramétrico de Mann-Whitney foi utilizado no pareamento casos (DMl com DC) e controles (só DMl) e para verificar a existência de diferenças na distribuição destes grupos em relação às variáveis independentes de interesse (HbAlc, dose de insulina, tireoidopatia, $\mathrm{z}$ de peso e estatura, sintomas de DC, antecedentes familiais de DAI). O nível de significância assumido foi de $5 \%$.

\section{RESULTADOS}

Dos 195 pacientes que preenchiam os critérios de inclusão, 16 foram excluídos por não terem comparecido à coleta de sangue e três por terem o material hemolisado em duas ou mais coletas. Também foram excluí- dos cinco pacientes $(2,8 \%)$ com IgA indetectável (abaixo de $23,5 \mathrm{mg} / \mathrm{dL}$ ) pela possibilidade de apresentarem deficiência seletiva de IgA, o que poderia determinar resultado falso-negativo no EMA. Tinham diagnóstico prévio de DC duas pacientes, mas foram mantidas na casuística. Logo, 171 pacientes participaram do estudo, sendo $91(53,2 \%)$ meninas.

A média de idade ao diagnóstico do DMl foi de 6,4 $\pm 3,5$ anos $(0,3$ a 14,2$)$ e ao iniciar o seguimento no Ambulatório de 7,0 $\pm 3,4$ anos $(0,3$ a 14,2). No momento de realização do EMA, os pacientes apresentavam média de idade de $14,1 \pm 4,9$ anos ( 3,3 a 26,7$)$ e duração média de diabetes de 7,8 $\pm 3,9$ anos $(0,8$ a 18,9).

Em relação ao controle metabólico do diabetes, a média das $\mathrm{HbAlc}$ no ano anterior à realização deste estudo foi de $10,4 \% \pm 2,1 \%(6,4$ a 17,1$)$ para o valor de referência de $3,9 \%$ a $6,1 \%$, e a dose média de insulina utilizada de $0,87 \pm 0,22 \mathrm{UI} / \mathrm{kg} /$ dia $(0,17$ a 1,58$)$.

As tireoidopatias, com ou sem alteração funcional, estavam presentes em 45 pacientes (26,3\%): 12 eutireóideos com anticorpo antiperoxidase (AcTPO) positivo, 15 hipotireóideos com AcTPO negativo, 17 hipotireóideos com AcTPO positivo, e um com hipertireoidismo e AcTPO positivo. Outras quatro DAI foram encontradas em quatro pacientes $(2,3 \%)$ : vitiligo, espondiloartropatia, psoríase e hepatite auto-imune.

Em relação à ocorrência de DAI entre os parentes de primeiro grau obteve-se esta informação de 167 pacientes (três não conseguiram informar com precisão e uma era filha adotiva). Uma vez que cinco pares de irmãos fazem parte desta casuística, 162 famílias foram avaliadas. Também entre os familiares, a tireoidopatia foi a mais prevalente, embora não se possa afirmar que todos os casos tenham origem auto-imune. Foram relatados 22 casos $(13,6 \%)$, um em cada família, porquanto em 18 deles a mãe era o familiar afetado. O DMl esteve presente em 16 parentes $(9,9 \%)$, porquanto 14 deles irmãos e, destes, 11 fazem parte desta casuística. Encontrou-se, ainda, 2,4\% de colagenoses auto-imunes (duas mães com lúpus eritematoso sistêmico e duas mães com artrite reumatóide). Um dos pacientes é irmão de uma das pacientes com diagnóstico prévio de DC $(0,06 \%)$.

A busca por sintomas sugestivos de DC demonstrou que 62 pacientes $(36,3 \%)$ foram assintomáticos, $91(53,2 \%)$ com ao menos um sintoma do trato digestório e $15(8,8 \%)$ referiram ao menos um sintoma inespecífico. Entre os sintomas clássicos, a dor abdo- 
minal foi a queixa predominante, sendo referida por $38,7 \%$ dos pacientes, mas a distensão abdominal, a constipação e a epigastralgia também foram comuns $(34,9 \%, 24,5 \%, 20,7 \%$, respectivamente). Episódios freqüentes de diarréia foram relatados por $12,2 \%$ destes pacientes. Apenas quatro $(3,7 \%)$ referiram vômitos intermitentes e um $(0,9 \%)$ queixou-se de flatulência permanente. A artralgia foi o sintoma inespecífico mais citado (29,2\%). Queixaram-se de fadiga 12 pacientes $(11,3 \%)$ e cinco $(4,7 \%)$ referiram cefaléia. Nenhum paciente apresentou crise convulsiva que não fosse desencadeada por episódio de hipoglicemia.
Dos 171 pacientes avaliados, nove apresentaram EMA positivo (soroprevalência de $5,3 \%$ ), incluindo as duas pacientes com diagnóstico prévio de DC (pacientes 1 e 2 Tabela 1). Todos foram submetidos à biópsia intestinal, que confirmou o diagnóstico em sete casos, pois apresentavam alterações histológicas compatíveis com DC em atividade (Marsh tipo 3). Entre estes casos, duas irmãs (pacientes 6 e 7 - Tabela 1) e as pacientes 1 e 2 (Tabela 1), sabidamente celíacas, que deveriam estar em dieta isenta de glúten, a positividade do EMA demonstrou a não aderência ao tratamento. Sendo assim, a prevalência da associação entre DMl e DC encontrada foi de $4 \%$ e o valor preditivo positivo do EMA foi de $77,8 \%$.

Tabela 1. Caracterização dos pacientes diabéticos com anticorpo antiendomísio positivo.

\begin{tabular}{cccccccccccc}
\hline Caso & Gênero & Idade & tDM1 & $\mathbf{z} \mathbf{P}$ & $\mathbf{z E}$ & Insulina & HbAlc & Sintomas & Tireoidopatia & DAI & DC \\
\hline 1 & F & 0,6 & 9,4 & 0,25 & $-0,12$ & 0,68 & 9,4 & Clássicos & Hipot. Ac (-) & $(+)$ & Ativa \\
2 & F & 12,3 & 9,8 &,- 58 & $-1,90$ & 1,10 & 8,1 & Clássicos & Hipot. Ac (+) & $(-)$ & Ativa \\
3 & M & 6,3 & 10,3 & $-1,92$ & $-1,12$ & 1,09 & 10,0 & Clássicos & $(-)$ & $(+)$ & Ativa \\
4 & F & 9,4 & 12,5 & 1,48 & 0,41 & 0,70 & 9,9 & Clássicos & Hipot. Ac (+) & $(-)$ & Ativa \\
5 & M & 3,2 & 6,5 & $-0,30$ & $-1,03$ & 0,70 & 12,0 & Clássicos & $(-)$ & $(-)$ & Ativa \\
6 & F & 8,8 & 1,6 & $-2,12$ & $-1,62$ & 0,66 & 11,0 & Clássicos & $(-)$ & $(+)$ & Ativa \\
7 & F & 5,0 & 1,5 & $-1,41$ & $-2,38$ & 0,67 & 11,2 & Clássicos & $(-)$ & $(+)$ & Ativa \\
8 & M & 10,2 & 2,3 & $-1,26$ & $-0,53$ & 0,83 & 8,1 & $(-)$ & $(-)$ & $(-)$ & Ausente \\
9 & M & 7,0 & 2,2 & 1,15 & 0,22 & 0,87 & 7,3 & Clássicos & Hipot. Ac $(-)$ & $(-)$ & Ausente \\
\hline
\end{tabular}

$\mathrm{DM} 1$ = diabetes melito tipo $1:$ ldade $=$ idade em anos de diagnóstico de $\mathrm{DMl}: \mathrm{tDMl}=$ tempo em anos de duração do $\mathrm{DMl} ; \mathrm{z} \mathrm{P}=\mathrm{z}$ escore do peso: $\mathrm{E}=\mathrm{z}$ escore da estatura; insulina = dose de insulina em UI $/ \mathrm{kg} / \mathrm{dia} ; \mathrm{DAl}$ = antecedentes familiais de doenças auto-imunes; $\mathrm{DC}=$ doença celíaca; $\mathrm{F}=$ feminino; $\mathrm{M}=\mathrm{masculino} ; \mathrm{Hipot}$. = hipotireoidismo; $\mathrm{Ac}=$ anticorpo antiperoxidase e/ou antitireoglobulina; $(+)=$ presente; $(-)=$ ausente

A Tabela 2 apresenta as características clínicas dos grupos após pareamento entre casos (DMl-DC) e controles (DMl). Observou-se diferença apenas no número de pacientes com sintomas do trato digestório, que foi significativamente maior no grupo casos em relação aos controles $(\mathrm{p}=0,02)$. As demais variáveis avaliadas não apresentaram diferença significativa entre os casos e os controles.

Tabela 2. Aspectos clínicos e laboratoriais de pacientes com associação diabetes e doença celíaca em comparação a pacientes apenas com diabetes.

\begin{tabular}{|c|c|c|c|c|c|}
\hline & \multicolumn{2}{|c|}{$\mathrm{DM} 1+\mathrm{DC}(\mathrm{n}=7)$} & \multicolumn{2}{|c|}{ DM1 $(n=21)$} & \multirow[b]{2}{*}{$p$} \\
\hline & Média — DP & Variaçāo & Média \pm DP & Variação & \\
\hline$z$ peso & $0,66 \pm 1,28$ & $-2,12$ a 1,48 & $-0,39 \pm 0,97$ & $-2,60$ a 1,42 & 0,58 \\
\hline$z$ estatura & $-1,13 \pm 0,98$ & $-2,38$ a 0,41 & $-0,81 \pm 0,73$ & $-2,32$ a 0,80 & 0,30 \\
\hline Insulina (UI/kg/dia) & $0,80 \pm 0,20$ & 0,66 a 1,10 & $0,89 \pm 0,16$ & 0,55 a 1,14 & 0,12 \\
\hline \multirow[t]{2}{*}{ HbAlc (\%) } & $10,23 \pm 1,29$ & 8,10 a 12,0 & $9,97 \pm 1,94$ & 6,50 a 14,90 & 0,61 \\
\hline & \multicolumn{2}{|c|}{$\mathbf{n}$} & \multicolumn{2}{|c|}{$\mathrm{n}$} & \\
\hline Sintomas & \multicolumn{2}{|c|}{7} & \multicolumn{2}{|c|}{11} & 0,02 \\
\hline Tireoidopatia & \multicolumn{2}{|c|}{3} & \multicolumn{2}{|c|}{6} & 0,49 \\
\hline AfDAl & \multicolumn{2}{|c|}{4} & \multicolumn{2}{|c|}{4} & 0,06 \\
\hline
\end{tabular}

$\mathrm{DMl}=$ diabetes melito tipo 1; DC = doença celíaca; AfDAl = antecedentes familiais de doenças auto-imunes. 


\section{DISCUSSÃo}

Considerando-se a heterogeneidade clínica da DC e as dificuldades diagnósticas subseqüentes, o emprego da triagem sorológica facilitou a investigação desta doença na população em geral, sobremaneira entre os pacientes considerados de risco, como os diabéticos. Entre os marcadores sorológicos disponíveis, tanto o EMA quanto o anticorpo antitransglutaminase tecidual (anti-tTG) apresentam sensibilidade e especificidade elevadas, amplamente empregados nos programas de triagem, além de demonstrarem alta sensibilidade no monitoramento da adesão à dieta isenta de glúten, indispensável ao tratamento do paciente celíaco (12). Apesar de a praticidade (dosado por ELISA), nem todos os kits comerciais de anticorpo anti-tTG, disponíveis no mercado, apresentam sensibilidade e especificidade padronizadas por estudos multicêntricos (13). No entanto, em particular, a utilização do EMA demanda alguns cuidados, como garantir que o paciente não apresente deficiência seletiva de $\operatorname{IgA}$ (o EMA é um anticorpo de classe IgA), não ser pesquisado em menores de 2 anos de idade, em virtude de sua baixa sensibilidade nesta faixa etária e dispor de profissional tecnicamente habilitado na detecção do EMA por imunofluorescência indireta, método semiquantitativo e observador dependente (12).

À semelhança do que ocorre em alguns países europeus, os estudos publicados no Brasil (6-9), ainda que escassos, têm demonstrado tendência de variabilidade regional da prevalência da associação entre DMl e DC, de $2,6 \%$ (7) a $15,8 \%$ (9), provavelmente influenciada pela intensa miscigenação do povo brasileiro, mas que também deve sofrer interferência de fatores ambientais e da dieta, como tempo de aleitamento, idade de introdução do glúten e composição da dieta, além da sensibilidade e especificidade do método empregado.

No presente estudo encontrou-se prevalência de DC semelhante à descrita em estudos realizados na Europa e nos Estados Unidos (14-16).

Pacientes inicialmente considerados assintomáticos, quando interrogados, retrospectivamente, sobre a presença de sintomas gastrintestinais, a resposta foi afirmativa. No presente estudo, todos os pacientes com DC associada referiram apresentar ou terem apresentado em algum momento os sintomas clássicos desta doença e, quando comparados a seus controles apenas diabéticos, a presença destes sintomas foi significativamente mais freqüente, com predomínio de dor abdominal. Bytzer e cols. (17) ao submeteram 145 pacientes com DMl ao Diabetes Bowel Symptom Questionnaire, instrumento de pesquisa de sintomas digestivos em pacientes diabéticos, observaram que $42 \%$ deles apresentavam pelo menos um dos sintomas pesquisados. Entretanto, nenhum destes pacientes foi triado para DC ou qualquer outra doença do trato digestório.

Há fortes evidências relacionando estas queixas às alterações do sistema neuroendócrino intestinal do paciente diabético mal compensado (18). No entanto, esta condição deve ser considerada um diagnóstico de exclusão, principalmente em pacientes pediátricos, nos quais outras doenças merecem ser pesquisadas, como as parasitoses, a doença de Hirschprung, as síndromes malabsortivas decorrentes de intolerâncias alimentares, entre outras.

O pareamento de casos (DMl e DC) e controles (só $\mathrm{DMl}$ ) não permitiu identificar qualquer outro parâmetro clínico (peso, estatura, necessidade de insulina ou valor de $\mathrm{HbAlc}$ ) que sugerisse interferência da DC no controle metabólico do diabetes. Ainda que a baixa estatura seja descrita como único sinal clínico em até 10\% dos celíacos (3), e que alguns estudos tenham descrito incidência maior de baixa estatura em pacientes com associação de DMl e DC quando comparados a pacientes apenas diabéticos $(19,20)$, o presente estudo, a exemplo de outros (21-23), não observou esta associação.

Da mesma forma, não existe consenso quanto à influência da DC na necessidade de insulina e nível de HbAlc. Segundo Barera e cols. (19), a presença de DC não interfere nestas variáveis. Kaspers e cols. (20) também não observaram diferenças quanto à dose de insulina utilizada, mas encontraram HbAlc significativamente menor no grupo com DC associada e atribuíram este fato à má-absorção decorrente da enteropatia celíaca. Medina e cols. (23) não encontraram diferenças no valor da HbAlC após a retirada do glúten em pacientes com a associação DC e DMl. O presente estudo foi pioneiro no Brasil em avaliar os aspectos clínicos da associação entre DMl e DC. Ainda que não se tenha encontrado indícios de influências da DC no controle metabólico do $\mathrm{DMl}$, este aspecto continua sendo muito controverso e mais estudos são necessários para que esta questão seja definitivamente esclarecida.

Em relação à associação com outras DAI, a tireoidopatia tem sido descrita como a mais prevalente (24) entre os diabéticos e o presente estudo pode comprovar isso. A associação entre DC e tireoidopatia auto-imune também é bem conhecida na literatura (25). Entretanto, no presente estudo, ao comparar a prevalência de 
tireoidopatia auto-imune entre pacientes diabéticos com e sem DC associada, não foi observada diferença entre os grupos. É possível que o número de pacientes desta amostra tenha interferido neste resultado. Também se deve considerar o fato deste ser um estudo transversal, realizado em uma população jovem, e que a prevalência de anticorpos antitireoidianos aumenta com a idade (26), assim como pode haver soroconversão positiva do EMA ao longo do tempo (21).

Avaliando a prevalência de DAI, inclusive tireoidopatia, nos parentes de primeiro grau dos pacientes com $\mathrm{DMl}$ associado ou não a $\mathrm{DC}$, este estudo mostrou uma tendência do grupo com associação de DC apresentar potencial auto-imune superior. Um estudo multicêntrico realizado em Israel demonstrou que a presença de outra DAI associada ao DMl aumenta significativamente o risco de DAI entre os parentes de primeiro grau, sugerindo a existência de "auto-imunidade familial” (24). Novamente, é provável que o número de pacientes desta amostra tenha interferido neste resultado.

Portanto, a utilização da triagem sorológica contribuiu para aumentar a prevalência de DC neste grupo de pacientes com DMl, justificando sua inclusão na rotina de seguimento destes pacientes e o EMA demonstrou ser uma ferramenta sensível, tanto no diagnóstico quanto no monitoramento da adesão à dieta isenta de glúten. Segundo o guideline da North American Society for Pediatric Gastroenterology, Hepatology and Nutrition (NASPGAN), a triagem deve ser periódica, com repetição quando houver sintomas, e, em especial, nos que apresentem HLA DQ2 ou DQ8 (13). Crone e cols. (21) observaram soroconversão para DC com biópsia positiva até oito anos após a primeira triagem, o que faz Cerutti e cols. (27) recomendarem a triagem anual até dez anos após o início do diabetes, quando parece haver redução da prevalência da DC. No entanto, outros autores sugerem a triagem anual de DC nos primeiros três a cinco anos após o diagnóstico de DMl, e a cada três a cinco anos após ou quando houver sintomas (28). Embora a forma clássica da DC tenha predominado nesta casuística, é importante ressaltar que os sintomas foram mencionados apenas após questionamento direcionado. A forma clássica é rara entre os pacientes diabéticos e os sintomas, quando presentes, podem ser subestimados por médicos e pacientes, fato que reforça a importância da realização da triagem. Não há ainda consenso na literatura a respeito da freqüência da realização da triagem para DC entre pacientes com DMl.

\section{AGRADECIMENTOS}

À Marise Mello Carnelossi Brunelli, técnica do Laboratório de Gastroenterologia Pediátrica da Unicamp pelo seu empenho na execução da imunofluorescência indireta para pesquisa do EMA. À Dra. Maria de Fátima Servidoni, gastroenterologista infantil, pela realização das endoscopias e à Dra Luciana Rodrigues de Meirelles, patologista clínica do Gastrocentro da Unicamp, pela análise histológica das biópsias.

\section{REFERÊNCIAS}

1. Sperling MA. Diabetes mellitus. In: Sperling MA, editor. Pediatric endocrinology. $2^{\mathrm{a}}$ ed. Philadelphia: Saunders; 2002. p. 323-6.

2. Schober E, Rami B, Granditsch G, Crone J. Coeliac disease in children and adolescents with type 1 diabetes mellitus: to screen or not, to treat or not? Horm Res. 2002;57 Suppl 1:97-100.

3. Hill ID, Bhatnagar S, Cameron DJ, De Rosa S, Mäki M, Russell GJ, et al. Celiac disease: working group report of the first world congress of Pediatric Gastroenterology, Hepatology and Nutrition. J Pediatr Gastroenterol Nutr. 2002;35 Suppl 2:S78-88.

4. Mäki M, Mustalahti K, Kokkonen J, Kulmala P, Haapalahti M, Karttunen T, et al. Prevalence of celiac disease among children in Finland. N Engl J Med. 2003;348(25):2517-24.

5. Collin $P$, Kaukinen K, Välimäki M, Salmi J. Endocrinological disorders and celiac disease. Endocr Rev. 2002;23(4):464-83.

6. Baptista ML, Koda YK, Mitsunori R, Nisihara, loshii SO. Prevalence of celiac disease in Brazilian children and adolescents with type 1 diabetes mellitus. J Pediatr Gastroenterol Nutr. 2005;41(5):621-4.

7. Tanure MG, Silva IN, Bahia M, Penna FJ. Prevalence of celiac disease in Brazilian children with type 1 diabetes mellitus. J Pediatr Gastroenterol Nutr. 2006;42(2):155-9.

8. Araújo J, Silva GA, Melo FM. Serum prevalence of celiac disease in children and adolescents with type 1 diabetes mellitus. J Pediatr (Rio J). 2006;82(3):210-4.

9. Brandt KG, Silva GA, Antunes MM. Celiac disease in a group of children and adolescents with type 1 diabetes mellitus. Arq Bras Endocrinol Metab. 2004;48(6):823-7.

10. Holmes GK. Non-malignant complications of coeliac disease. Acta Paediatr. 1996;412(Suppl):68-75.

11. Marsh MN. Gluten, major histocompatibility complex, and the small intestine: a molecular and immunobiologic approach to the spectrum of gluten sensitivity ("celiac sprue"). Gastroenterology. 1992;102:330-54.

12. Collin $P$, Kaukinen $K$, Vogelsang $H$, Korponay-Szabó I, Sommer $\mathrm{R}$, Schreier $\mathrm{E}$, et al. Antiendomysial and antihuman recombinant tissue transglutaminase antibodies in the diagnosis of coeliac disease: a biopsy-proven European multicentre study. Eur J Gastroenterol Hepatol. 2005;17:85-91.

13. Hill ID, Dirks MH, Liptak GS, Colletti RB, Fasano A, Guandalini $S$, et al. Guideline for the diagnosis and treatment of coeliac disease in children: recommendations of the North American Society for Pediatric Gastroenterology, Hepatology and Nutrition. J Pediatr Gastroenterol Nutr. 2005:40:1-9. 
14. Barera G, Bonfanti R, Viscardi M, Bazzigaluppi E, Calori G, Meschi $F$, et al. Occurrence of celiac disease after onset of type 1 diabetes mellitus: a 6-year prospective longitudinal study. Pediatrics. 2002;109(5):833-8.

15. Aktay AN, Lee PC, Kumar V, Parton E, Wyatt DT, Werlin SL. The prevalence and clinical characteristics of celiac disease in juvenile diabetes in Wisconsin. J Pediatr Gastroenterol Nutr. 2001;33(4):462-5.

16. Poulain C, Johanet C, Delcroix C, Lévy-Marchal C, TubianaRufi N. Prevalence and clinical features of celiac disease in 950 children with type 1 diabetes in France. Diabetes Metab. 2007; 33:453-8.

17. Bytzer P, Talley NJ, Hammer J, Young LJ, Jones MP, Horowitz M. GI symptoms in diabetes mellitus are associated with both poor glycemic control and diabetic complications. Am J Gastroenterol. 2002;97(3):604-11.

18. El-Salhy, M. The possible role of the gut neuroendocrine system in diabetes gastroenteropathy. Histol Histopathol. 2002 17(4):1153-61.

19. Barera G, Bianchi C, Cerutti F, Dammacco F, Frezza E, Illeni MT, et al. Screening of diabetic children for coeliac disease with antigliadin antibodies and HLA typing. Arch Dis Child. 1991; 66(4):491-4.

20. Kaspers S, Kordonouri O, Schober E, Grabert M, Hauffa BP Holl RW; German Working Group for Pediatric Diabetology. Anthropometry, metabolic control, and thyroid autoimmunity in type 1 diabetes with celiac disease: A multicenter survey. $J$ Pediatr. 2004;145(6):790-5.

21. Crone J, Rami B, Huber WD, Granditsch G, Schober E. Prevalence of celiac disease and follow-up of EMA in children and adolescents with type 1 diabetes mellitus. J Pediatr Gastroenterol Nutr. 2003;37(1):67-71

22. Mohn A, Cerruto M, lafusco D, Prisco F, Tumini S, Stoppoloni $\mathrm{O}$, et al. Celiac disease in children and adolescents with type 1 diabetes: importance of hypoglycemic. J Pediatr Gastroenterol Nutr. 2001;32(1):37-40.

23. Medina YN, López-Capapé M, Orejas EL, Blanco MA, Salces CC, Castellanos RB. Impacto del diagnóstico de la enfermedad celíaca en el control metabólico de la diabetes tipo 1. An Pediatr (Barc). 2008;68:13-7.

24. Hanukoglu A, Mizrachi A, Dalal I, Admoni O, Rakover Y, Bistritzer $Z$, et al. Extrapancreatic autoimmune manifestations in type 1 diabetes patients and their first-degree relatives: a multicenter study. Diabetes Care. 2003;26(4):1235-40.

25. Toscano V, Conti FG, Anastasi E, Mariani P, Tiberti C, Poggi M, et al. Importance of gluten in the induction of endocrine au toantibodies and organ dysfunction in adolescents celiac patients. Am J Gastroenterol. 2000;95(7):1742-8.

26. Jaeger C, Hatziagelaki E, Petzoldt R, Bretzel RG. Comparative analysis of organ-specific autoantibodies and celiac diseaseassociated antibodies in type 1 diabetic patients, their firstdegree relatives, and healthy control subjects. Diabetes Care. 2001;24(1):27-32.

27. Cerutti F, Bruno G, Chiarelli F, Lorini R, Meschi F, Sacchetti C. Younger age at onset and sex predict celiac disease in children and adolescents with type 1 diabetes mellitus: an Italian multicentre study. Diabetes Care. 2004;27:1294-8.

28. Schwarzenberg SJ, Brunzell C. Type 1 diabetes and celiac disease: an overview and medical nutrition therapy. Diabetes Spect. 2002;15:197-201

\section{Endereço para correspondência:}

Gil Guerra-Júnior

Departamento de Pediatria

CP 6111 FCM - Unicamp

13083-100 Campinas, SP

E-mail: gilguer@fcm.unicamp.br 